J. Lake Sci. (湖泊科学), 2013, 25(2): 171-187

http://www. jlakes. org. E-mail : jlakes@niglas.ac.cn

(C) 2013 by Journal of Lake Sciences

\title{
单巢类轮虫有性生殖的研究进展与展望”
}

孙 栋, 牛翠娟**

(北京师范大学生命科学学院生物多样性与生态工程教育部重点实验室, 北京 100875)

\begin{abstract}
摘 要: 作为轮虫动物门的主体, 单巢类轮虫是淡水浮游动物的重要组成部分. 它们通常作为一类主要的初级消费者在 淡水生态系统中起着重要作用, 因而深人了解它们在自然水体中的种群动态及其决定机制对淡水生态系统理论与应用 研究都有重要意义. 作为一类具有典型周期性孤雌生殖生活史的动物, 有性生殖的发生和进化策略对其种群动态和进化 都有重要影响, 因而单巢类轮虫有性生殖的机理和进化研究一直是轮虫学研究领域的一个核心内容. 本文就当前单巢类 轮虫有性生殖的主要研究进展,包括其发生机理、信息素、理论模型、影响因素和相关实证研究等进行了系统综述; 特别 就单巢类轮虫有性生殖策略的三个理论模型: “bang-bang” 模型、中等有性生殖比率模型和非延迟的有性生殖模型进行了 详细论述. 此外, 本文还对影响单巢类有性生殖的内外源因素进行了总结, 主要的外源因素包括食物的浓度及种类、温 度、盐度和特殊的细菌等, 而主要的内源性因素则包括母体年龄、食物浓度诱导的跨代母系效应、延迟的有性生殖和其他 一些潜在的母系效应因素等. 其中, 因为延迟的有性生殖现象广泛存在且可能是轮虫物种共存或克隆共存现象的一种潜 在解释, 所以就此现象的理论模型、机理和实证研究等方面进行了深人讨论. 另外, 本文还简要讨论性比率资源分配与单 巢类轮虫有性生殖的关系. 最后, 对未来几个可能的热点方向简单评述, 以期为国内轮虫学和相关方面的研究提供帮助.

关键词: 浮游动物; 单巢类轮虫; 周期性孤雌生殖; 有性生殖; 种群动态; 理论模型; 性比率; 有性生殖延迟
\end{abstract}

\section{Sexual reproduction in monogonont rotifers: A review}

\author{
SUN Dong \& NIU Cuijuan \\ (Ministry of Education Key Laboratory for Biodiversity Science and Ecological Engineering, College of Life Sciences, Beijing \\ Normal University, Beijing 100875, P. R. China)
}

\begin{abstract}
This paper focuses on the recent findings of sexual reproduction in monogonont rotifers, which account for the main bulk of phylum Rotifera. Generally, they act as one of the major groups of primary consumers in freshwater ecosystem. Thus, studies on population dynamics and their mechanisms of monogonont rotifers may provide important data to both theoretical and applied research on freshwater ecosystems. As a group with parthenogenetic life history, sexual reproductive strategy in monogonont rotifers may profoundly affect their population dynamics and evolution. This review summarized several aspects of sexual reproduction in the monogonont rotifers, such as the mechanism, pheromones, models, influence factors, and particularly, empirical evidences. More specifically, we emphasized three models about their sexual reproduction patterns: "bang-bang” model, intermediate mictic ratios model and no-delaying mixis model. Then we summarized the exogenous and endogenous factors that affect sexual reproduction. The major exogenous factors include the concentration and type of food, temperature, salinity and special bacteria, while the major endogenous factors include the maternal age, a transgenerational maternal effect induced by food concentration, mixis delay and some potential maternal effects. We especially highlighted the model, mechanism and empirical evidences of mixis delay, since this phenomenon existed widely and was deem to be a potential explanation for species or clones coexistence. We also gave some personal views on the relationship between sex allocation theory and sexual reproduction in the monogonont rotifers. Finally, several potential hotspots in the future study were highlighted.
\end{abstract}

Keywords: Zooplankton; monogonont rotifers; cyclical parthenogenesis; sexual reproduction; population dynamics; models ; sex allocation; mixis delay

* 国家自然科学基金项目(30970349)资助. 2012-04-13 收稿;2012-06-19 收修改稿. 孙栋,男,1986 年生,博士研 究生; E-mail:bio. sundong@ 126. com.

** 通信作者;E-mail : cjniu@ bnu. edu. cn. 
轮虫是一类体型微小的后生动物, 通常体长 $100 \sim 1000 \mu \mathrm{m}$, 目前已知约 2000 种, 隶属轮虫动物门 (Rotifera) ${ }^{[1]}$. 一般认为, 轮虫动物门包括 3 大类群: 单巢纲 (Monogononta) (1570 种)、蛭态纲 (Bdelloidea) (461 种) 和尾盘纲 (Seisonidea) (3 种) ${ }^{[2]}$. 轮虫动物门 3 个主要类群的生殖方式迥异, 单巢纲为典型的周期 性孤雌生殖 (cyclical parthenogenesis), 蛭态纲为无性的孤雌生殖 (asexual parthenogenesis), 而尾盘纲为有性 生殖 (sexual reproduction). 作为轮虫动物门的主体, 单巢类轮虫广泛分布于河湖、沼泽和海洋等各类水体, 特 别是淡水浮游动物的重要组成部分, 在河流和湖泊生态系统的物质循环、能量流动和信息传递中起着关键 作用. 研究单巢类轮虫在自然水体中的种群动态及其决定机制对淡水生态系统管理具有重要的理论和应用 价值. 作为周期性孤雌生殖的动物, 有性生殖的发生和进化对策对单巢类轮虫种群动态和进化都有着根本 性的影响, 因而一直是轮虫学研究的核心内容. 本文就单巢类轮虫有性生殖的最新研究进展做一系统综述, 以期为国内相关领域的研究提供参考.

\section{1 单巢类轮虫有性生殖的机理}

\section{1 轮虫有性生殖的信息素}

在单巢类轮虫中, 很多证据表明它们使用一些化学信号来调节其生殖方式 (即孤雌生殖到有性生殖的 转变). 早在 $1960 \mathrm{~s}$, Gilbert ${ }^{[3]}$ 就证实莒花臂尾轮虫 (Brachionus calyciflorus) 和角突臂尾轮虫 (B. angularis) 种 群由无性繁殖到有性繁殖的转变是由种群自身释放到水体中的一种化学物质所诱发的. Snell 等 ${ }^{[4]}$ 证明 B. manjavacas (属于褶铍臂尾轮虫 (B. plicatilis) 种复合体) 有性生殖的触发是由一种其自身释放的 $39 \mathrm{kDa}$ 的 蛋白质 (被定义为“有性生殖诱导蛋白”, MIP) 在水体中积累所致. 进一步的研究发现单巢类轮虫有性生殖 触发过程与细菌中的群体感应现象 (quorum sensing) 很相似 ${ }^{[5]}$. 轮虫有性生殖的另一个重要环节一一交配行 为, 也是由化学信号触发的. 目前已经确定在轮虫身体表面存在一种特殊的糖蛋白, 被称为 “雄性识别信息 素” (MRP), 当雄虫与雌虫相遇时, 这种糖蛋白通过两者的身体直接接触而被体表的性信息素感受器所探 知, 并完成交配前的物种识别和确认过程 ${ }^{[6-7]}$. 最近的研究表明 MRP 基因属于一个编码一类相似蛋白质的 基因家族,因而它可能是单巢类轮虫中存在快速进化现象的一种潜在解释 ${ }^{[8-9]}$.

到目前为止, 还没有任何一种轮虫的信息素被准确鉴定 (解析出其晶体结构), 但有大量证据表明单巢 类轮虫种群的混交雌体比率可对多种动物的信息素做出反应. 这些物质包括脊椎动物的生长激素 (vertebrate growth hormone)、孕酮 (progesterone)、人体线毛膜促性腺激素 ( human chorionic gonadotropin)、 $17 \beta$-雌二醇 (17 $\beta$-estradiol) 和三碘甲状腺氨酸 (triiodothyronine) 以及昆虫中的类固醇二十差基蜕皮酮 (steroid 20-hydroxyecdysone) 和保幼激素 (juvenile hormone) ${ }^{[10-12]}$. 尽管上述研究都没有准确测定轮虫体内的被试 物质含量与培养液中此物质含量的关系, 而且培养液中的被试物质浓度可能与轮虫体内此物质的浓度差异 极大, 同时这类物质在轮虫体内的吸收和代谢途径也远未明晰, 但考虑到这些物质的水溶性特征和在自然 界中分布的广泛性, 这些实验结果都暗示某种类固醇类 ( steroid) 的内源性物质可能在单巢类轮虫有性生殖 的调控过程中起着核心作用.

另外, 研究证实某些药物、杀虫剂和有机化合物可对水生动物内分泌系统造成干扰 ${ }^{[13]}$. 在单巢类轮虫 中, 人们也发现水体中常见的内分泌干扰物质, 如壬基苯酚 (nonylphenol)、睪酮 (testosterone)、氟他胺 (flutamide)、早熟素 (precocene)、抗雄性激素杀蛽松 (anti-androgens fenitrothion) 和环丙氯地孕酮 (cyproterone acetate) 等都可显著影响颌花臂尾轮虫的有性生殖和休眠卵生产 ${ }^{[14-15]}$. 这些实验证据启示单巢类轮虫的有性 生殖对于雄激素及其拮抗物质干扰的敏感度可能要高于雌激素类物质的干扰, 同时也进一步支持类固醇类 物质可能是单巢类轮虫有性生殖过程关键信息素的观点.

\section{2 轮虫卵母细胞的发育过程及外源物质影响其发育的信号通路}

在很多动物中, 卵母细胞的发育过程都受到各种信号转导通路的紧密调控 ${ }^{[16]}$. 但在轮虫中, 调控卵母细 胞发育过程的信号转导通路至今尚未完全阐明. 在轮虫中卵母细胞的成熟是连续性的, 而卵黄腺产生的卵 黄物质开始注人到卵细胞中标志着这一系列过程的起始 ${ }^{[17]}$. 近期研究表明, 母体产出后代的生殖类型 (混 交雌体或是非混交雌体) 在其产卵前较短时间内才被确定, 大约是卵细胞在卵巢内发育的时段 ${ }^{[18]}$; 同时研 究发现当母体暴露于含有一定浓度孕酮的培养液中时, 后代的生殖类型会受到影响 ${ }^{[12]}$. 这暗示通过抑制某 
些卵母细胞发育信号通路上关键基因的表达就可以人为改变它们后代的生殖类型 ${ }^{[19]}$.

目前认为单巢类轮虫卵母细胞发育过程的内分泌信号有两种可能的通路:一种可能的途径是体外的 有性生殖诱导信号穿越轮虫的体壁, 直接作用于卵母细胞, 并导致卵母细胞发育过程中的特定基因表达 发生改变, 从而产生混交雌体. 具体来说, 这种途径中对有性生殖诱导信号的受体可能直接存在于卵母细 胞表面, 也可能存在于卵黄腺, 通过卵母细胞发育过程中卵黄物质的注人而将信息间接传递给卵母细 胞, 也可能两者皆有. 另一种可能是卵母细胞和卵黄腺都没有信号受体, 或者体外的有性生殖诱导信 号无法穿透轮虫体细胞到达生殖腺, 而受体存在于轮虫体细胞 (更可能是存在于体表). 体细胞的受体 接受信息后通过神经系统或是其他体内的信息传递方式将有性生殖的诱导信号传递至生殖腺和生殖 细胞 ${ }^{[20]}$.

\section{2 单巢类轮虫有性生殖发生的诱导因素}

到目前为止, 已经有 3 种环境因子被证实可诱导单巢类轮虫的孤雌生殖母体产生有性后代, 进一步诱 导种群发生有性生殖. 这 3 种环境因子包括种群密度信号、食物中的维生素 $\mathrm{E}$ 和长的光周期.

\section{1 种群密度信号}

密度效应 (crowding effect) 是单巢类轮虫中研究最为广泛同时也是最早发现的一种有性生殖诱导信 ${ }^{{ }^{5}}{ }^{[21]}$. 实验室研究在褶皱臂尾轮虫种复合体 ${ }^{[4,22-24]}$ 、蕶花臂尾轮虫 ${ }^{[25-26]}$ 、角突臂尾轮虫、臂尾水轮虫 (Epiphanes brachionus)、椎尾水轮虫 (E. senta)、前额犀轮虫 (Rhinoglena frontalis) ${ }^{[27]}$ 和颤动疮毛轮虫 (Synchaeta tremula $)^{[28]}$ 中都发现高的种群密度可以诱导有性母体的产生. 同时野外观察的证据也支持这个结论 ${ }^{[29]}$. 在 其他周期性孤雌生殖的浮游动物 (如枝角类) 中, 也有很多证据表明高的种群密度可以诱导种群发生有性生 殖 ${ }^{[30-31]}$. 如此广泛的实证表明, 高种群密度会诱导种群进行有性生殖在整个单巢类轮虫中可能是一种普遍 现象.

近期研究证实在禇皱臂尾轮虫中这种诱导种群发生有性生殖的物质是一种 $39 \mathrm{kDa}$ 的蛋白质 ${ }^{[4]}$. 尽管在 其他轮虫种类中有性生殖诱导信号物质并未得到深人研究, 但考虑到有性生殖诱导信号所必须具备的种间 特异性 (见下文论述) 和保守性特征, 其他的单巢类轮虫很可能也是采用类似蛋白质作为诱导信号 (MIP) ${ }^{[4,32]}$. 在自然水体中, 某一特定轮虫物种的 MIP 浓度取决于轮虫种群释放积累速率和水体中细菌分解 速率的平衡. 其中轮虫释放 MIP 的速率主要取决于轮虫种群大小、年龄结构和种群增长率, 水体中 MIP 分解 的速率则主要与温度和细菌群落构成有关 ${ }^{[32]}$. 最近的模型研究还认为轮虫种群释放 MIP 很可能存在一种 正反馈机制, 即水体中 MIP 的积累会促进轮虫种群加快释放 MIP 的速率 ${ }^{[33]}$, 但是更为确丵的实验证据目前 仍然很缺乏.

\section{2 食物中的维生素 $\mathrm{E}$}

关于食物中的维生素 $\mathrm{E}$ 诱导轮虫发生有性生殖的研究集中于晶囊轮虫属 (Asplanchna) 的几个种类, 其 中包括卜氏晶囊轮虫 (Asplanchna brightwelli)、间型晶囊轮虫 (A. intermedia) 和西氏晶囊轮虫 ( A. sieboldi). 当孤雌繁殖的晶囊轮虫母体摄人富含维生素 $\mathrm{E}$ (即 $\alpha$-生育酚) 的食物时, 它们就可以生产混交后代 ${ }^{[34-35]}$. 事 实上, 维生素 $\mathrm{E}$ 很可能是通过直接控制晶囊轮虫非混交雌体的多态性 (一种晶囊轮虫特有的体型大小和身 体结构发生显著改变的现象, 并非诱导防御现象) 而影响后代的生殖方式. 关于食物中维生素 $\mathrm{E}$ 诱导轮虫产 生有性生殖后代的生态意义, Gilbert 进行了详细讨论: 在自然水体中维生素 $\mathrm{E}$ 只能由绿藻和高等植物合成, 因而捕食性的晶囊轮虫只有通过摄食植食性轮虫 (例如臂尾轮虫和龟甲轮虫等) 才可能摄人充足维生素 $\mathrm{E}$. 有研究发现富含维生素 $\mathrm{E}$ 的食物可以诱导晶囊轮虫的体型和结构发生显著变化: 孤䧳生殖的雌体体型增 大, 同时具有显著宽阔的头冠部 (称为 “钟型”, campanulate morphotype), 这些体型和结构的变化有利于晶囊 轮虫进一步扩大食物范围, 使得它们能够捕食较大的轮虫和甲壳类的猎物 ${ }^{[32,35]}$. 高的维生素 $\mathrm{E}$ 摄人量意味 着环境中食物丰富 (尤其是可以为晶囊轮虫提供丰富营养的植食性轮虫), 随着种群密度上升, 雌雄个体的 相遇概率也增大, 同时丰富的食物可以允许受精雌体为休眠卵提供更为丰富的营养储备 (主要是脂类物

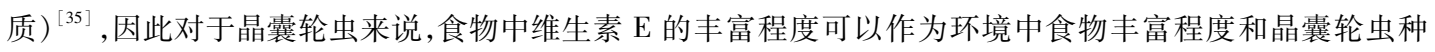
群密度的良好指示. 


\section{3 长的光周期}

到目前为止人们仅在椎轮属 (Notommata) 和异尾轮属 (Trichocerca) 中发现长的光周期可诱导孤雌生殖 种群产生混交个体 ${ }^{[36-37]}$. 光周期作为一种信号的特点是高度的稳定性和可预测性. 当轮虫将光周期与环境 条件及种群密度联系时, 长的光周期意味着合适的水温和环境条件以及随之而来的种群密度上升, 这些都 符合资源需求假说和雌雄相遇假说 (两个假说内容见下文论述). 但是光周期作为有性生殖诱导因素的基本 前提是轮虫生活环境中的各种条件 (特别是食物丰富程度以及随之而来的种群密度) 与光周期强烈而且稳 定地关联, 这点在臂尾轮虫属和晶囊轮虫属显然是不具备的, 因而这些类群的轮虫只有依靠与自身所处环 境条件更为直接相关的因素 (例如种群密度) 而不是极端稳定和可预测的光周期来作为有性生殖的诱导 因素 ${ }^{[32,38]}$.

\section{3 有性生殖诱导信号的物种特异性}

衡量周期性孤雌生殖轮虫长期适合度的合理指标是一个生长季内休眠卵的生产量 ${ }^{[21]}$, 而在较高的种群 密度下进行有性生殖有利于雌雄个体的相遇和交配, 因而有助于提高休眠卵产量. 如果同域共存的多个轮 虫物种的有性生殖诱导信号没有种间界限, 那么先萌发的物种在水体中积累的 MIP 就会让后萌发的物种在 种群密度仍然较低时面临高的 MIP 浓度 (从而触发种群的有性生殖). 而此时如果后萌发物种的种群被触发 有性生殖, 那么此物种的种群增长率将受到极大抑制, 同时产生的休眠卵总数量也会较少. 因而理论上说, 在温带水体中分布的单巢类轮虫, 其有性生殖诱导信号 (MIP) 应该存在显著的种间隔离机制, 即 MIP 的物 种特异性 ${ }^{[39]}$. 但迄今为止只有少数研究探讨了有性生殖诱导信号在浮游动物中的种特异性问题, 主要集中 于枝角类水蚤属和单巢类轮虫臂尾轮属 ${ }^{[40-41]}$.

近期有两个更为系统的实验探究了臂尾轮虫类的有性生殖诱导信号物种特异性的问题. Gilbert ${ }^{[25]}$ 发现 颌花臂尾轮虫北美品系和澳洲品系个体释放的 MIP 都可以诱导同品系其他个体产生有性个体, 但是不能诱 导对方品系个体产生有性个体. 这表明荅花臂尾轮虫的有性生殖诱导存在分类上 (地理品系间) 的特异性. 后续的分子系统学和交配实验研究表明, 澳洲品系很可能是苛花臂尾轮虫种复合体内的一个隐种 ${ }^{[42]}$. Stelzer 等 ${ }^{[43]}$ 利用核糖体 ITS1 序列构建了褶皱臂尾轮虫种复合体内 5 个不同地理隐种间的系统发育树, 并与它 们相互之间的有性生殖诱导特异性进行了比较. 结果并未发现种复合体内的地理隐种间在有性生殖诱导 方面存在特异性, 而且也与系统发育关系无关. 为什么在蕶花臂尾轮虫种复合体和禇皱臂尾轮虫种复合 体内隐种间得到的结论完全不同? 目前尚无实验证据或理论假说很好解释此差异. 不过有实验证据表明 在单巢类轮虫的部分类群 (包括颌花臂尾轮虫和福皱臂尾轮虫) 存在有性生殖延迟现象 (详见下文论 述) ${ }^{[27,44]}$ 和交配行为上的生殖隔离 ${ }^{[42,45]}$, 这可能是这种不依赖于有性生殖诱导信号种间特异性的隐种共 存的一种解释.

\section{4 关于单巢类轮虫有性生殖发生时刻的假说}

实验室和野外研究都发现单巢类轮虫有性生殖的发生时刻及种群中有性个体的比率通常都与种群密 度紧密相关 ${ }^{[24,29]}$. 关于单巢类轮虫种群有性生殖的发生, 目前主要有 3 个假说.

\section{1 环境恶化假说 ( habitat deterioration hypothesis)}

环境恶化假说认为由于周期性孤雌生殖动物有性生殖的发生可以显著抑制种群的增长 ${ }^{[46]}$, 因而在环境 条件适宜时它们进行孤䧳生殖快速扩大克隆种群, 而有性生殖应该在环境条件恶化时发生 ${ }^{[47]}$. 在环境恶化 时进行孤雌生殖产生休眠卵有两个优势: 一是可以借助休眠卵的形式渡过不良环境条件; 二是有性生殖和 基因重组带来的遗传上的多样性有利于种群在未来的生长季适应新的环境.

\section{2 雌雄相遇假说 (male-female encounter hypothesis)}

雌雄相遇假说的前提是雌雄个体在水体中的相遇是随机的, 随着种群密度的增加, 这种相遇的概率和 受精的成功率也随之增大 ${ }^{[48]}$, 这样可以保证克隆种群产生尽量多的休眠卵. 如果种群在低密度下即大量进 行有性生殖, 不仅受精成功率低, 而且还要显著牺牲种群在一个生长季内的增长潜力和竞争能力 (均依赖孤 雌生殖) ${ }^{[21,49]}$. 


\section{3 资源需求假说 (resource-demanding hypothesis)}

在自然环境中, 种群密度较高的时期通常也与食物条件适宜的时期, 在此时种群进行有性生殖除了可 以有利于雌雄相遇之外, 丰富的食物还有利于受精雌体产生数量较多且质量较好的休眠卵 (其中储存的脂

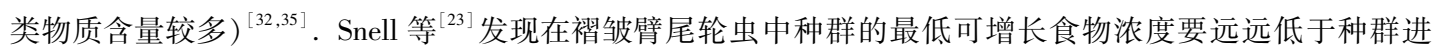
行有性生殖所需的最低食物浓度. 而最近关于颌花臂尾轮虫休眠卵生产与环境食物条件关系的研究中, 下 列两个假说得到证实: 第一, 混交雌体生产休眠卵所需要的最低食物浓度要远高于非混交雌体生产非混交 卵的食物需求; 第二, 当环境中食物浓度较低时, 混交雌体生产休眠卵的数量较少 (净生殖率低), 只有当食 物条件适宜时混交雌体休眠卵的生产量才能够达到最大值 ${ }^{[50]}$. 上述研究都表明单巢类轮虫成功进行有性生 殖并产下正常的休眠卵所需要的食物条件要远高于种群增长所需要的食物阈值, 即从食物资源的角度来说 有性生殖和休眠卵生产只能在食物条件适宜的环境中发生.

\section{5 单巢类轮虫有性生殖发生的几个理论模型}

周期性孤雌生殖动物 (例如单巢类轮虫) 有性生殖的发生对于其种群增长有着显著的代价 ${ }^{[46,51]}$. 孤雌生 殖带来的种群快速增长可提高某一物种或基因型的竞争能力, 在捕食风险存在的环境中这一点更为重要. 而有性生殖产生的休眠卵又是种群渡过不良环境所必须的形式, 因此休眠卵产量是某一物种或基因型的长 期适合度指标 ${ }^{[21]}$. 在周期性孤䧳生殖动物的生活史中, 一个核心问题是种群如何权衡孤雌生殖和有性生殖.

很多单巢类轮虫都生活于不同程度的暂时性生境中. 对于不同物种来说, 每一个可利用生长季节的长 度从几天到几个月不等. 例如, 对分布于北美洲 Chihuahuan 沙漠中的一种六腕轮虫 Hexarthra sp. 来说, 由于 沙漠中降雨很少且很快蒸发, 因而它们的每一个生长季节就只有几天, 而且长度和间隔都高度不可预测 ${ }^{[52]}$. 而对于温带淡水湖泊中常见的颌花臂尾轮虫, 其每一个生长季可长达几个月, 而且年际变化不大. 正是基于 轮虫生境的暂时性特征, Serra 等 ${ }^{[51]}$ 提出了轮虫种群有性生殖发生的 “bang-bang” 策略模型 (图 1a). 此模型 认为, 周期性孤雌生殖轮虫种群的有性生殖存在一个 0 或 1 的策略, 即当某一生长季内轮虫种群达到一个较 高的阈密度 (图 1a 中的 “有性生殖起始” 密度) 后种群只会生产有性个体, 而有性生殖对当前的种群增长没 有贡献, 同时由于个体的自然死亡和被捕食造成的种群密度下降会让种群达到一个较低的阈密度 (图 1a 中 的“有性生殖终止” 密度), 此时有性生殖完全消失, 种群回到完全的孤雌生殖方式以快速增殖, 随后进人下 一个“有性生殖起始/终止”周期.

a

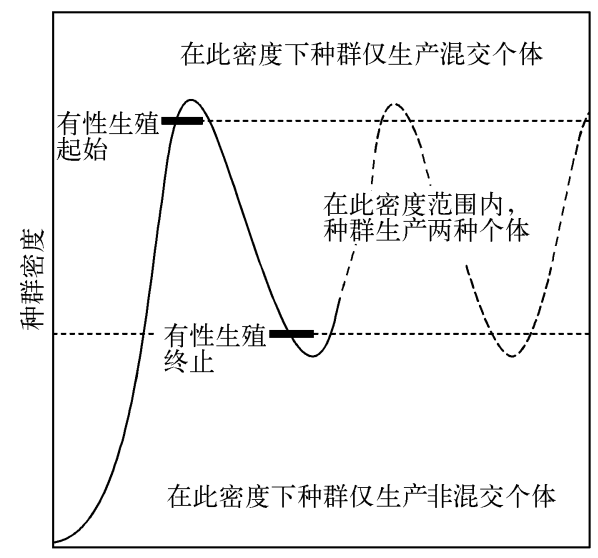

时间 $\mathrm{b}$

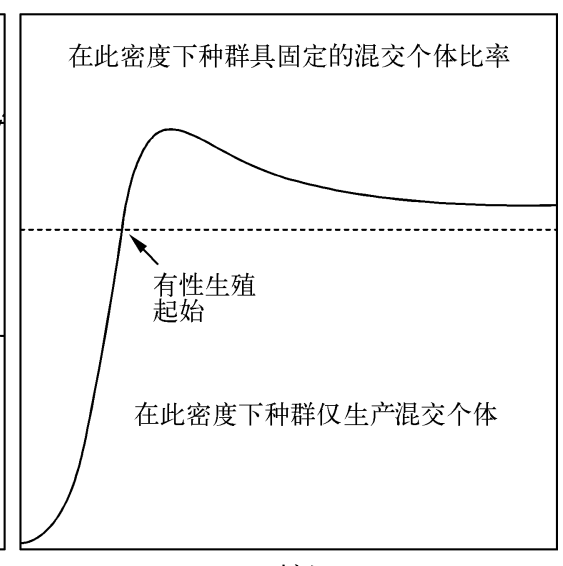

时间

图 1 单巢类轮虫有性生殖的理论模型 (a: “bang-bang” 策略模型; b: 中等有性生殖比率模型) ${ }^{[21]}$

Fig. 1 Two models of sexual reproduction in the monogonont rotifers (a: "bang-bang" model; b: intermediate mictic ratios model) 
理论模型的模拟还发现在这种有性生殖模式下有性生殖的最佳时刻 (即生长季内总的休眠卵产量最 高) 是种群的死亡率刚刚超过出生率之时, 这时种群中新生的个体将全部为有性个体, 直到种群密度低于 “有性生殖终止” 密度. 如果自然水体中的轮虫种群也是采取这种 “bang-bang” 策略, 那么如果连续监测的话 应该可以观察到某一时段出现极高的有性个体比率, 但是野外观察数据并不支持这种“bang-bang”策略. 例 如, Carmona 等 ${ }^{[29]}$ 在 1992 年 7 月到 1993 年 11 月间连续监测了西班牙地中海沿岸 3 个小型咸水湖中禇皱臂 尾轮虫种群的动态, 发现多数种群在大部分时间采取以孤雌生殖为主的繁殖方式, 所有样本中有性个体的 比率为 $0 \sim 29 \%$ 之间. 最高 $29 \%$ 的有性个体比率显然并不支持上述的 “bang-bang” 策略模式. 实验室内关于 轮虫种群生态学的研究也发现在各种培养条件下轮虫种群中有性个体的比率一般不超过 $50 \%{ }^{[40,53]}$. 上述 野外观察和室内实验的结果表明, 温带湖泊中单巢类轮虫的有性生殖采取此种策略模式的可能性并 不大.

自然水体中进行的食物添加实验证实温带湖泊中的轮虫种群经常处于食物限制的环境中 ${ }^{[54]}$, 因此在一 个生长季节内轮虫的种群增长通常是密度依赖性的. 基于轮虫这种密度依赖性的种群增长模式, Serra 等 ${ }^{[21]}$ 提出了一个密度依赖性轮虫种群的最佳有性个体比率模型：

$$
m_{\mathrm{o}}=1-(q / b)^{1 / 2}
$$

式中, $m$ 为有性个体比率, 种群中有性雌体数量与种群中总雌体数量的比值; 其中, 此模型中假定在一个生 长季内, 某一特定克隆种群的有性个体比率为一恒定值; $m_{\mathrm{o}}$ 为最佳有性个体比率, 在此有性个体比率下, 种 群一个生长季内的休眠卵产量最高; $q$ 为种群的死亡率; $b$ 为种群的出生率.

对于一个特定的克隆种群, 当环境条件好转时, 死亡率下降、出生率上升, 此时种群的最佳有性个体比 率上升; 反之, 当环境恶化时 (例如种群的快速增长导致的食物缺乏), 死亡率上升、出生率下降, 种群的最佳 有性个体比率随之下降. 自然水体中的轮虫种群出生率通常大于死亡率 (至少在整个生长季节的大部分时 间是这样), 根据上述模型, 轮虫种群应采取中等的有性生殖比率, 而不是 “bang-bang” 策略模型的 0 或 1 比 率. 这一结论也与早期 Snell 模型的模拟结果相吻合: 根据 13 个禇皱臂尾轮虫地理品系的生命表实验数据 ${ }^{[55]}$ 修正了模型中的种群增长率参数后, 此模型模拟出福皱臂尾轮虫种群的最佳有性生殖比率在 $10 \% \sim 50 \%$ 之 间; 而综合多个实验结果得到的禇皱臂尾轮虫种群平均有性个体比率为 $21.20 \% \pm 3.84 \%$ (变化范围为 $0 \sim 50 \%)^{[46]}$.

这个模型的前提假设就从另一方面解释了为什么 “bang-bang” 策略模型预测的有性生殖模式很难在实 际观察中发现: 由于通常温带水域分布的单巢类轮虫生活于食物受限制的环境中 ${ }^{[54]}$, 而种群在这种相对稳 定和食物作为主要限制因子的环境中显然是一种密度依赖性的增长 (即有一个食物条件决定的环境容量 $\mathrm{K}$ ), 此时轮虫种群更倾向于采取中等的有性生殖比率 (图 1b) ; 只有当种群长期处于一种高度可预测的临时 性环境, 同时种群在生长季内的主要限制因子不是食物而是像灾变性的干旱等突发性限制因子时, “bangbang”有性生殖模式似乎才有可能在轮虫种群中被保留下来 ${ }^{[21]}$.

多个轮虫有性生殖发生的模型都发现有性生殖对种群增长有着显著的代价 ${ }^{[21,46]}$. Serra 等 ${ }^{[21]}$ 认为也可 以从有性生殖的代价角度解释为什么环境条件适宜时种群的最佳有性个体比率会上升:

$$
R C_{\mathrm{m}}=b \cdot m /(b-q)
$$

式中, $R C_{\mathrm{m}}$ 为种群有性生殖的相对代价 (relative cost of mixis), $m$ 为有性个体比率, $q$ 为种群的死亡率, $b$ 为种 群的出生率.

模型的说明: $b \cdot m$ 可以视为种群进行有性生殖对种群增长的绝对代价, $b-q$ 可以认为是没有有性生殖 时种群的增长率, 因而 $b \cdot m /(b-q)$ 即为有性生殖对种群增长的相对代价. 如果种群的有性个体比率不变, 当环境条件好转时, $b$ 上升、 $q$ 下降, 有性生殖的相对代价下降, 这里计算的种群有性生殖的相对代价是针对 短期适合度的, 由于种群的短期适合度 (一个生长季内的种群增长) 和长期适合度 (生长季内的休眠卵产 量) 存在着权衡关系 ${ }^{[46,49]}$, 因此当种群有性生殖导致的短期适合度代价下降时, 选择压力将会偏好种群提高 有性生殖比率以获得更高的长期适合度. 
表 1 环境的可预测性与轮虫种群的有性生殖策略*

Tab. 1 The relationship between the environmental predictability and the sexual reproduction strategy in the monogonont rotifers

\begin{tabular}{|c|c|c|c|}
\hline 基本模型 & “bang-bang”模型 $[51]$ & 非延迟的有性生殖模型 [21,39] & 中等有性生殖比率模型 ${ }^{[21]}$ \\
\hline 环境的可预测性 & 可预测的,变化的环境 & 高度不可预测的、随机变化的环境 & 可预测的、较稳定的环境 \\
\hline $\begin{array}{l}\text { 种群的有性生殖 } \\
\text { 比率 }\end{array}$ & 高, 接近 1 & $\begin{array}{l}\text { 通常在种群密度低时较低, 随着种 } \\
\text { 群增长逐渐变为中等的 }\end{array}$ & $\begin{array}{l}\text { 中等的, 通常小于或约等于 } \\
0.5\end{array}$ \\
\hline $\begin{array}{l}\text { 有性生殖的发生 } \\
\text { 时刻 }\end{array}$ & $\begin{array}{l}\text { 种群增长到最大密度,环境 } \\
\text { 条件开始不适宜时 }\end{array}$ & $\begin{array}{l}\text { 种群增长的早期即开始有性生殖, } \\
\text { 不论此时环境条件是否适宜 }\end{array}$ & $\begin{array}{l}\text { 种群增长达到环境容纳量, } \\
\text { 环境条件通常仍适宜种群 } \\
\text { 增长 }\end{array}$ \\
\hline $\begin{array}{l}\text { 是否是密度依赖 } \\
\text { 的种群增长 }\end{array}$ & 非密度依赖的 & 非密度依赖的 & 密度依赖的 \\
\hline 有性生殖阈密度 & 通常较高 & $\begin{array}{l}\text { 生长季早期极低, 随着种群增长逐 } \\
\text { 渐升高 }\end{array}$ & 通常较高 \\
\hline 野外实例 & $\begin{array}{l}\text { 西班牙地中海沿岸小型咸 } \\
\text { 水湖泊中褶皱臂尾轮虫的 } \\
\text { 冬季种群 }\end{array}$ & $\begin{array}{l}\text { 北美洲 Chihuahuan 沙漠中的一种 } \\
\text { 六腕轮虫 Hexarthra sp. }{ }^{[52]}\end{array}$ & $\begin{array}{l}\text { 典型温带湖泊 Golf Course } \\
\text { Pond 中的盖氏晶囊轮虫 } \\
\text { A. girodi }{ }^{[56]}\end{array}$ \\
\hline
\end{tabular}

* 非延迟的有性生殖模型详见下文关于“延迟的有性生殖” 部分的论述.

\section{6 影响单巢类轮虫有性生殖的主要外在因素}

人们在长期的研究中还发现, 有一些环境因子虽然不能够直接诱导轮虫发生有性生殖, 但可以显著影 响轮虫的有性生殖策略, 如改变种群的有性生殖阈密度等. 目前已知的主要外在因素有食物、温度、盐度、特 殊的细菌等.

\section{1 食物}

单巢类轮虫有性生殖对食物条件的要求要显著高于孤䧳生殖. 如 Snell 等 ${ }^{[57-58]}$ 发现食物种类可显著影 响褶坡臂尾轮虫的休眠卵和雄性个体生产: 适宜环境下饲喂禇坡臂尾轮虫普通小球藻 Chlorella vulgaris 和 Baker's 酵母混合的食物时, 其休眠卵和雄性个体的产量显著高于饲喂其相同碳浓度的一种蓝藻 Schizotrix calcicola 时的产量; 研究的同时发现轮虫种群进行有性生殖需要更高的食物浓度: 当培养液中盐藻Dunaliella 的浓度为 $1.53 \times 10^{4} \mathrm{cells} / \mathrm{ml}$ 时, 轮虫有性个体生产就会停止, 但孤雌生殖直到培养液中盐藻浓度低至 $1.50 \times 10^{3} \mathrm{cells} / \mathrm{ml}$ 时才会停止 ${ }^{[23]}$. 在荳花臂尾轮虫中也发现类似的现象. 当培养液中的啮蚀隐藻 (Cryptomonas erosa var. reflexa) 浓度为 $1.25 \times 10^{3}$ cells $/ \mathrm{ml}$ (极低的食物浓度) 时, 孤雌生殖个体的净生殖率为 1.4 , 而有性生殖个体产生休眠卵的净生殖率仅为 $0.9^{[50]}$.

\section{2 温度}

一些室内实验发现温度可影响轮虫种群中有性个体的比率, 但这种影响并不具有一致性. 例如, Hagiwara 等 ${ }^{[59]}$ 发现培养温度为 $15^{\circ} \mathrm{C}$ 时禇皱臂尾轮虫种群的有性个体比率要高于培养温度为 $20 、 25$ 或 $30^{\circ} \mathrm{C}$ 时的 种群, 这似乎可以解释为低温下种群的有性生殖阈密度更低; 然而在臂尾水轮虫中发现了相反的趋势 ${ }^{[60]}$. 面 临环境温度变化时, 这种有性生殖倾向性的种间差异可能与自然水体中此物种的种群动态与水温的相关性 有关. 假设在自然水体中, 上述实验中的轮虫在其一个生长季中于水温 $15^{\circ} \mathrm{C}$ 左右时, 其最适食物种类的密度 最高, 由于有性生殖和生产休眠卵需要更多的能量, 因此此时是种群进行有性生殖的最佳时刻. 如果这种食 物条件和环境温度的相关性是相对稳定的, 即种群可以通过水温来预测环境中的食物条件, 那么长期的选 择压力就有可能导致这个种群在 $15^{\circ} \mathrm{C}$ 下更倾向于进行有性生殖.

\section{3 盐度}

水体盐度是影响很多咸水轮虫生活史与分布的重要因子. 近期研究表明咸水湖泊中的盐度异质性很可 能是禇皱臂尾轮虫种复合体内隐种同域共存现象的一个重要原因 ${ }^{[61]}$. 大量研究证实盐度对轮虫的有性生殖 
也有显著影响. 例如, Lubzens 等 ${ }^{[62]}$ 发现褶皱臂尾轮虫种群在盐度为 4\% ～20\% 的培养环境中种群的有性个 体比率最高, 盐度低于或高于此范围时有性个体比率急剧降低, 表明某些盐度范围有利于有性生殖发生. Snell ${ }^{[63]}$ 关于环境对禇皱臂尾轮虫种群有性生殖净生殖率影响的研究也发现了类似规律, 并且认为相比于孤 雌生殖, 有性生殖更易受环境限制. 褶䏢臂尾轮虫种复合体内的不同隐种有不同盐度偏好 ${ }^{[61]}$, 而且在不同盐 度下的有性生殖倾向性也有显著差别. 如 Hagiwara 等 ${ }^{[64]}$ 发现禇皱臂尾轮虫的 “ $L ”$ 型种群 (事实为一个隐种) 在 $8 \%$ 的低盐度环境中更倾向于有性生殖, 随着水体盐度的升高, 其有性生殖受到显著抑制; 而 “S” 型种群 (另一个隐种) 的反应与之相反, 其在 $32 \%$ 的高盐度环境中更倾向于有性生殖, 随着水体盐度的降低, 其有性 生殖受到显著抑制.

\section{4 特殊的细菌}

关于水体中细菌直接影响轮虫有性生殖的研究很少, 目前只有 Hagiwara 等 ${ }^{[65]}$ 研究了水体中人为添加 特定细菌对禇皱臂尾轮虫有性生殖的影响. 研究发现当水体中添加少量假单胞菌 (Pseudomonas)、莫拉氏菌 (Moraxella) 或微球菌 (Micrococcus) 时, 褶皱臂尾轮虫实验种群的有性个体比率相比于对照组上升了 $4 \sim 10$ 倍. 尽管目前对该现象尚无确切的解释, 但猜测可能是由于这些细菌向水体中释放了结构上类似于可诱导 种群发生有性生殖的轮虫密度信号类的代谢物质, 从而极大提高了种群的有性个体比率. 此外, 水体中的细 菌对轮虫的有性生殖可能还有一种潜在的间接影响: 在自然水体中, 单巢类轮虫有性生殖诱导信号 (即种群 密度信号) 的积累是一个轮虫种群向水体中不断释放与水体中的细菌不断分解之间的平衡过程 ${ }^{[32]}$, 因而特 定细菌群落的存在可能加速或减慢这种分解过程, 进而改变水体中轮虫有性生殖信号的积累速率, 并间接 对有性生殖造成影响. 考虑到自然淡水水体中细菌所占的巨大生物量 ${ }^{[66]}$, 关于水细菌的群落结构和代谢活 动对轮虫自然种群有性生殖的影响尚需进一步研究.

\section{7 影响单巢类轮虫有性生殖的主要内在因素}

关于影响单巢类轮虫有性生殖内在因素的研究远不如外在因素的研究广泛, 到目前为止只有母体年龄 (即后代的出生顺序效应) 的影响、食物因素导致的泛代母系效应的影响和有性生殖延迟 (一种泛代表型可 塑性) 等三个主要方面得到实验证实.

\section{1 母体年龄}

早在 $1940 \mathrm{~s}$, 人们就在轮虫中发现母体年龄 (即后代的出生顺序) 的多代积累效应可以对后代的一些生 活史特征造成持续的影响 ${ }^{[67]}$, 后来的研究进一步证实这种现象在轮虫中是普遍存在的 ${ }^{[68-69]}$. 近期的研究表 明母体年龄还可以影响轮虫后代的棘刺长度和休眠卵的形态及孵化率等 ${ }^{[70-71]}$.

在单巢类轮虫中, 有实验证据表明母体有随年龄增长, 所产生有性后代比例降低的倾向. 目前已知符合

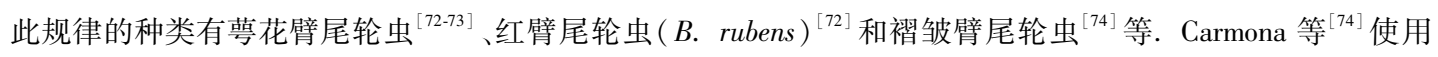
克隆种群的禇皱臂尾轮虫自交得到一些休眠卵, 然后分别孵化并各自建立新的克隆种群, 结果发现这些克 隆种群在有性生殖倾向性方面差别显著, 由年轻母体生产的休眠卵所建立的克隆种群中有性个体比率高于 年老母体生产的休眠卵所建立的克隆种群, 表明休眠卵产生时的母体年龄对后代的有性生殖策略产生了显 著影响. 但是后来的研究还发现单巢类轮虫后代有性生殖倾向性与母体年龄的这种规律并不像之前认为的 那样稳定, 在一些禇皱臂尾轮虫和颌花臂尾轮虫品系中后代有性生殖倾向性与母体年龄无关 ${ }^{[75-76]}$. Lubzens 等 ${ }^{[77]}$ 报道在其研究的福皱臂尾轮虫品系中母体繁殖中期生产的后代表现出最大的有性生殖倾向性. 在一些 䓵花臂尾轮虫品系中也发现此现象 ${ }^{[78]}$.

上述的大量研究证据表明单巢类轮虫的母体年龄与后代有性生殖倾向性之间的关系存在高度的多样 性, 这种多样性存在于种间、品系间和克隆间等不同水平. 目前关于这个方面还有以下几点尚不清楚: 第一, 单巢类轮虫表现出的这种高度多样性是由基因决定还是依赖于环境条件亦或是两者的共同作用? 第二, 如 果是基因决定的, 则是由细胞核基因决定还是细胞质遗传 (母系遗传)? 第三, 如果证实是依赖于环境条件 的,那么这种多样性的适应意义是什么? 关于上述方面还有待进一步的实验证明.

\section{2 食物因素导致的跨代母系效应 (transgenenrational maternal effect)}

从轮虫休眠卵中直接孵化出的雌体通常称为干雌体 (stem female), 它们具有一些适合繁殖高质量的后 
代以便快速建立克隆种群的生理特征, 例如身体具有显著较高的脂质含量等 ${ }^{[79]}$. Hino 等 $^{[53]}$ 发现改变禇皱 臂尾轮虫干雌体培养环境中的食物浓度可以对随后的整个克隆种群有性生殖的倾向性产生持续多代的影 响, 这可能是由于干雌体对环境中食物条件的变化更为敏感, 同时环境变化对干雌体的影响可以通过母体 的细胞质遗传给后代, 从而产生多代的持续效应. Hagiwara 等 ${ }^{[80]}$ 也发现褶皱臂尾轮虫干雌体生命早期经历 的饥饿环境 (饥饿 $12 \mathrm{~h}$, 对照组为不经历饥饿环境) 可以显著提高其后代的有性生殖倾向性, 并且这种影响 可以在第 8 代之后变得极为显著; 与此相比, 从非混交卵中孵化出的孤雌生殖雌体经历相同的饥饿环境却 不能够产生持续多代的显著的母系效应. 关于食物因素导致的泛代母系效应对后代有性生殖策略的影响方 面, 目前的研究仍然很缺乏, 进一步的研究应该更加关注以下方面: 一, 这种现象目前仅在禇䏢臂尾轮虫中 得到证实, 在其他轮虫, 特别是在休眠卵萌发时期常常面临水体中低食物浓度的淡水植食性轮虫 (如颌花臂 尾轮虫) 中是否也有此现象; 二, 从模型和实证上揭示这种现象的适应意义.

\section{3 延迟的有性生殖一一种跨代表型可塑性}

早在 $1970 \mathrm{~s}$, 人们在研究禇皱臂尾轮虫种群有性生殖时就发现随着从休眠卵中孵化出个体传代数的增 加, 非混交雌体产生混交雌体后代的倾向性增加, 即种群更倾向于进行有性生殖 ${ }^{[53]}$. 后来, Gilbert ${ }^{[4]}$ 详细研 究了颌花臂尾轮虫一个 Florida 品系的有性生殖策略后确定在一些克隆中的确存在这种规律, 并认为这对于 温带水域中生长季长度有限的单巢类轮虫是一种进化适应策略. 下面本文将从理论模型和实验证实两个方 面详细讨论这个现象.

7.3.1 延迟的有性生殖: 理论模型以密度依赖型种群增长时的有性生殖策略模型 ${ }^{[21]}$ 为基础, Serra 等 ${ }^{[39]}$ 发 表了一个可以模拟轮虫种群增长过程中存在有性生殖延迟现象时的有性生殖模型. 其模型中出生率、死亡 率和种群内禀增长率等参数均来自于禇䏢臂尾轮虫的研究结果. 限于本文的篇幅, 具体的推导过程这里不 再赘述. 这里笔者主要讨论一下基于实验和野外观察得到的参数而进行的模型模拟结果.

此模型的基本假定是生长季内存在多个萌发时刻不同的克隆, 同时克隆种群的有性生殖阈密度没有显 著差异, 且克隆间不存在有性生殖诱导信号 (MIP) 的特异性. 依据已有的研究结果, 在单巢类轮虫中上述 3 个前提假定是部分得到证实的 ${ }^{[43,81]}$. 在这种情况下, 一个生长季内较早萌发的克隆将迅速扩大种群并在水 体中积累 MIP, 而后萌发克隆的有性生殖诱导如果没有传代数的延迟, 则刚刚萌发在种群密度很低时即被水 体中已经存在 MIP 诱导发生有性生殖. 由于有性生殖对种群增长的显著代价 ${ }^{[21,46]}$, 后萌发克隆种群的短期 适合度将显著低于早萌发种群. 由于克隆种群的有性生殖发生在低种群密度下, 雌雄相遇的概率小, 因而有 性生殖的成功率低, 同时由于有性生殖发生在种群规模较小时, 因而总的休眠卵产量也较低 ${ }^{[46]}$, 这些都导致 后萌发克隆基因型的长期适合度较低. 相反, 如果后萌发克隆具有延迟的有性生殖策略, 那么就可以大大提 高基因型的短期适合度和长期适合度.

基于进化稳定对策 (ESS) 理论 ${ }^{[82]}$,Serra 等还模拟了具有延迟的有性生殖策略的基因型对不具有这种 策略基因型的人侵性 ${ }^{[39]}$. 侵人模型的研究发现: (1) 当具有此种策略的基因型的种群具有 $14 \%$ 的种群有性 个体比率和 $9 \mathrm{~d}$ 的有性生殖延迟时间时, 对不具有这种策略的种群人侵性最强; (2) 有性生殖延迟的时间短 于 $7 \mathrm{~d}$ 或超过 $11 \mathrm{~d}$ 都会对采取此策略基因型的适合度造成显著代价; (3) 某一基因型即使具有延迟的有性 生殖策略, 其长期的进化优势也很小, 概略的估计为经过 40 个生长季后, 具有 $9 \mathrm{~d}$ 最佳延迟时间策略的基因 型相比于不具有任何有性生殖延迟策略的基因型在基因型频率上的优势不会超过 0.05 , 因而同一水体中几 种具有不同策略的基因型是可以长期共存的.

关于 Serra 等 ${ }^{[39]}$ 模型和 ESS 可侵人性模型模拟结果,本文主要讨论下面几个问题:

第一, 模型模拟得出当具有此种策略的基因型的种群具有 $14 \%$ 的种群有性个体比率和 $9 \mathrm{~d}$ 的有性生殖 延迟时间时, 对不具有这种策略的种群人侵性最强. 而基于相同前提假设和模拟参数(种群为密度依赖型增 长和禇皱臂尾轮虫的种群增长数据) 的 Serra 等 ${ }^{[21]}$ 模型得出禇皱臂尾轮虫种群的最佳有性个体比率为 $45 \%$. 两个模型得出的最佳有性个体比率都相差极大, 对此 Serra 等给出的解释是早期的模型仅仅考虑到水 体中存在一个基因型的情况, 而 ESS 可侵人性模型则着重研究水体中存在多个基因型时的情况: 当不同基 因型的克隆种群同域共存时, 克隆种群间的竞争能力 (主要取决于孤雌繁殖方式的种群增长率) 是一个必须 要考虑的因素, 而某一基因型具有较高的有性个体比率 (如 Serra 和 King 模型中得出的 45\% ) 显然不利于其 
在一个生长季内基因型间的竞争能力. 相比较而言, ESS 可侵人性模型则综合考虑了生长季内不同克隆间 (基因型间) 的竞争能力和整个生长季内生产休眠卵的数量, 因而可能更为合理. 另外, 实证数据也更倾向于 支持 ESS 可侵人性模型: 综合多个有关禇皱臂尾轮虫实验结果和野外观测数据得出的最佳有性个体比率为 $21.20 \% \pm 3.84 \%^{[46]}$.

第二, 关于最佳的有性生殖延迟时间 (传代数), 当前模型得出在褟皱臂尾轮虫中大约为 $9 \mathrm{~d}$, 同时认为 超过 $11 \mathrm{~d}$ 时延迟的有性生殖带来的适合度优势将会迅速下降. 到目前为止并没有关于禇皱臂尾轮虫有性生 殖延迟时间 (或代数) 的实验数据, 而在同属的莒花臂尾轮虫一个 Florida 品系中, 轮虫从干雌体生出后第 5 8代的有性生殖倾向性 (约 10\% 20\% ) 要显著高于刚从干雌体中生出的第 1 代, 同时要显著低于 12 代之 后的种群(约 $40 \% \sim 50 \%)^{[44]}$, 这表明这个品系的颌花臂尾轮虫有性生殖延迟的代数(受到内源因素抑制的 代数) 不低于 8 代. 在 $20^{\circ} \mathrm{C}$ 食物适宜的环境中, 颌花臂尾轮虫通常 $36 \sim 48 \mathrm{~h}$ 一代 (笔者本人的观察), 因而这 里的有性生殖延迟的时间大约为 $12 \sim 16 \mathrm{~d}$. 考虑到禇皱臂尾轮虫具有更高的种群增长率 (适宜条件下其种 群增长率可达到 $1.0 \mathrm{~d}^{-1[83]}$ ), 因而上述模型模拟得出的最佳有性生殖延迟时间很可能是合理的.

第三, ESS 可侵人性模型模拟结果表明轮虫种群采取延迟的有性生殖策略可带来一定的适合度优势, 尽 管其长期进化优势很小 (40 个生长季后仅达到不超过 0.05 的基因型频率变化). 然而考虑到轮虫世代周期 很短且水域环境中种间及种内不同基因型间激烈的竞争, 很小的适合度劣势都可能导致物种或基因型的灭 绝. 实证表明这种有性生殖延迟策略在单巢类轮虫中尽管大量存在, 却并非是一种普遍存在的规律 ${ }^{[27,44]}$, 其 原因何在？对此笔者提出下面几个可能的解释.

1) 轮虫生活水域的环境 (主要是食物条件) 存在明显的年际间波动 (即存在波动的选择压力) ${ }^{[81]}$. 当某 一生长季内的食物条件非常适宜, 此时那些没有有性生殖延迟策略的种群(在种群增长早期即进行有性生 殖产生休眠卵) 由于具有较高的种群增长率, 因而有性生殖对种群增长的代价不大, 同时不同基因型种群间 的竞争也要比环境胁迫时弱很多, 所以这些基因型依旧可以达到较大的种群规模同时留下大量休眠卵. 考 虑到周期性孤雌生殖浮游动物休眠卵库普遍具有的 “储存效应” ( storage effect) ${ }^{[84]}$, 只要多个生长季内有其 中一个环境条件非常适宜, 那么不具有有性生殖延迟策略的基因型就将一直保持相当的比例而不至于灭绝 (尽管比例上有所波动).

2) 从轮虫休眠卵的萌发模式考虑, 上述模型的默认假设是轮虫休眠卵在极短时间内大量萌发, 即暴发 式萌发. 但事实上, 大量实验证据表明轮虫休眠卵的萌发模式在种间和种内不同克隆间 (基因型间) 均存在

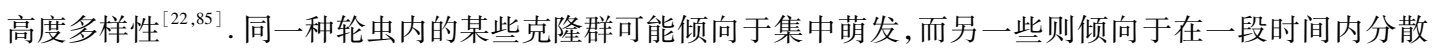
萌发. 例如, 在研究了海城地区一个淡水湖泊中蔩花臂尾轮虫休眠卵的萌发模式后, Liu 等 ${ }^{[85]}$ 发现同域共存 的不同克隆间休眠卵的萌发模式存在显著差别, 一些克隆的休眠卵在适宜环境中 $3 \mathrm{~d}$ 内的萌发率即达到或 接近 100\% (暴发式萌发), 而另一些则需要 $8 \mathrm{~d}$ 甚至更长时间 (分散式萌发). 由此可见上述模型的基本假设 就存在一些缺陷. 如果假定某一具有有性生殖延迟策略基因型的休眠卵采取分散式萌发, 而另外一个不具 有此策略基因型的休眠卵采取暴发式萌发, 而且假定最初触发它们萌发的环境条件一致, 那么在春季环境 条件逐渐好转达到触发萌发的条件时, 水体中后一基因型的种群规模将很快超过前一基因型的种群, 并且 大量占据水体中有限的资源, 从而在基因型间的竞争中占据优势地位. 因而如果考虑到休眠卵萌发模式的 复杂性, 单纯假定单一暴发式萌发而建立的 ESS 可侵人性模型可能并不能真正揭示轮虫是否采取延迟的有 性生殖策略的进化优势. 而关于轮虫不同物种间或基因型间是否表现延迟的有性生殖策略与它们休眠卵孵 化模式关联性的研究目前还没有报道, 这方面的实证研究对进一步评价单巢类轮虫延迟的有性生殖策略的 进化优势和解释种内不同克隆间在有性生殖策略上的多样性 ${ }^{[27]}$ 都有很重要的意义.

3 ) 轮虫在生长季内, 特别是在生长季早期面临的捕食压力和种间竞争压力的大小可能会对轮虫是否采 取延迟的有性生殖策略造成显著影响. 以捕食压力为例, 当生长季早期轮虫克隆种群面临的捕食压力较小 时, 其采取延迟的有性生殖策略可以避免或降低有性生殖对种群增长的代价, 从而保证轮虫种群面临一定 的捕食压力时仍然能够维持增长. 这样达到更高的种群密度后触发有性生殖可以提高雌雄相遇概率并生产 更多的休眠卵, 从而获得高的长期适合度. 另一方面, 当生长季早期轮虫克隆种群面临的捕食压力较高时, 仅靠降低有性生殖投资无法维持种群增长, 此时采取非延迟的有性生殖策略从而尽快产生较多休眠卵以保 
证某一基因型的种群渡过不良环境就更为有利. 如果多个不同基因型共存的一个自然水体中某一种轮虫在 生长季早期面临的捕食压力存在显著的年际间变化, 那么某一个生长季早期的捕食压力就很大程度上决定 了不同有性生殖延迟策略的基因型在这一个生长季是否具有适合度优势 ( 以休眠卵产量计算), 再考虑到休 眠卵库的储存效应, 在这种捕食压力存在显著年际间变化的水体中, 多个不同策略基因型的长期共存是完 全可能的. 但到目前为止关于单巢类轮虫有性生殖延迟与环境中捕食压力的关系尚缺乏实验证据.

4) 环境条件的可预测性与轮虫的有性生殖延迟策略之间的关系. ESS 可侵人性模型更多的是考虑在 一种稳定环境中的最佳对策, 即在高度可预测环境中的情况. 在这种情况下, 单巢类轮虫的有性生殖采取一 定时间的延迟是有利的, 但是如果环境条件高度不可预测, 例如在沙漠中靠聚集雨水形成的小池塘中生存的 轮虫种类, 如果仍然采取一定时间的有性生殖延迟, 那么就有可能面临环境条件快速恶化后 (如水体干涸) 有 性生殖尚未发生, 从而在一个生长季内无法产生休眠卵的情况. 考虑到轮虫的休眠卵通常采取 “两面下注” 的 萌发策略 ${ }^{[81]}$, 因而即使在一个生长季内种群无法产生休眠卵可能也不至于导致某一基因型在休眠卵库中完全 灭绝, 但经过一个或多个这样的生长季后, 整个休眠卵库中具有延迟策略的基因型频率将显著低于不具有这 种延迟策略的基因型频率. 因此从理论上说, 在这种高度不可预测环境中轮虫不应采取延迟的有性生殖策 略 ${ }^{[27]}$. 对于这个假说, 目前有两个实证研究的报道,下面本文着重讨论一下这两个研究的重要发现.

7.3.2 延迟的有性生殖与环境条件的可预测性: 实证研究 上面的理论分析表明单巢类轮虫是否采取延迟的 有性生殖策略与所处环境的可预测性相关, 因而人们期望从不同环境条件 (主要是生长季长度的可预测性不 同) 的自然水体中观察到采取不同对策的轮虫来验证此假说 (即 “在不可预测环境中, 轮虫不会采取延迟的有 性生殖策略” 假说). 目前主要有 3 个实验研究 (表 2):1) Gilbert ${ }^{[44]}$ 研究了一个荳花臂尾轮虫 Florida 品系从休 眠卵中孵化出的第 $1 、 5 、 8 、 12$ 和 18 代个体的有性生殖倾向性, 结果发现第 1 代个体的有性生殖倾向性极低; 第 5 代和第 8 代的有性个体比率平均值大约为 $10 \% \sim 20 \%$; 在第 12 代和第 18 代种群中有性生殖倾向性显著上 升, 大约为 $40 \% \sim 50 \%$. 这证实了在此品系轮虫中存在明显的有性生殖延迟策略, 而此品系最初采集于一个 临时性的湖泊. 因而作者认为此实验并不支持上述假说 ${ }^{[27]}$. 2) Schröder 等 ${ }^{[27]}$ 研究了不同环境中分布的一个 蘦花臂尾轮虫品系, 一个角突臂尾轮虫品系, 两个椎尾水轮虫品系和两个前额犀轮虫 (Rhinoglena frontalis) 品系的有性生殖延迟策略, 并比较了环境的可预测性 (永久性水体和临时性水体) 与轮虫采取的有性生殖延 迟策略间的关系, 发现大多数结果并不支持上述假说 (表 2). 3 ) 沙漠中依赖降雨而形成的临时性小水塘可 以被认为是一种极端不可预测的环境, 对于这种环境中生存的轮虫来说, 其生长季的长度可能短至仅有几 天到十几天的时间, 上述理论分析认为在这种环境中轮虫绝不应采取延迟的有性生殖策略. Schröder 等 ${ }^{[52]}$ 调查了北美洲 Chihuahuan 沙漠里小型临时性水塘中分布的一种六腕轮虫 Hexarthra sp. 的有性生殖模式后发 现它们从休眠卵中孵化出的干雌体即有 7\% 46\% 为混交雌体, 而在其他生活史已知的单巢类轮虫中通常干 雌体全部为非混交雌体 ${ }^{[79]}$. 在这种高度不可预测环境中的种类不具有任何延迟的有性生殖策略显然是支持 上述假说的.

表 2 显示大多数实验结果并不支持上述假说. 关于出现这种结果的原因, Schröder 等 ${ }^{[27]}$ 给出了两条解 释:1) 对于某一特定种类轮虫来说, 其临时性生境存在的时间 (即可利用的生长季长度) 稳定的超过此种轮 虫有性生殖延迟的时间, 即环境条件的不可预测性没有成为一种极端的限制因子. 例如, 他们研究的椎尾水 轮虫有性生殖延迟的时间通常为 $6 \sim 10$ 代, 种群完成这些世代大约需要 $4 \sim 5$ 周, 而奥德河洪水泛滥期 (即 季节性河漫滩生境中椎尾水轮虫的生长季) 往往要长于此时间, 因而在这种环境中延迟的有性生殖策略还 是具有适合度优势的;2) 在轮虫生活的自然水体中, 食物因子 (通常是各种微藻) 的变化幅度很大, 例如在 很多温带湖泊中夏季存在一个食物浓度很低的 “净水期” ( clear-water phase) ${ }^{[86]}$, 因而在所谓的 “永久性湖 泊” 中轮虫生长季的长度也是有限的, 甚至在极端情况下每个生长季的长度可能还要短于上述的各种“临时 性” 生境. 因而在永久性生境中, 轮虫克隆种群在种群增长早期 (即从休眠卵中孵化出后较少的传代数内) 即 进行有性生殖生产休眠卵也是对食物因素决定的(不可预测的)生长季长度的一种两面下注.

另外, 在这类实验中基本上均采取了标准密度信号诱导的方法测定克隆种群不同传代数个体的有性生 殖倾向性, 例如, 在上述第一个实验中采用了大约 $6 \mathrm{ind}$. $/ \mathrm{ml}$ 的种群密度作为标准密度信号环境, 如此高的 种群密度在永久性的自然水体中极为罕见 (尽管在一些高度临时性生境中可能存在 ${ }^{[1]}$. 因而在这种过高的 种群密度信号下得到的有性生殖延迟倾向性的实验结果可能与自然状态下种群的表现有所偏差. 
表 2 目前关于“在不可预测环境中,轮虫不会采取延迟的有性生殖策略”假说的实证研究*

Tab. 2 Empirical evidences for the hypothesis “rotifers do not have the mixis delay strategy in unpredictable environments"

\begin{tabular}{|c|c|c|c|c|}
\hline 物种 + 品系 & 采集环境 & 有性生殖延迟策略 & $\begin{array}{l}\text { 是否支持 } \\
\text { 上述假说 }\end{array}$ & $\begin{array}{l}\text { 参考 } \\
\text { 文献 }\end{array}$ \\
\hline 墓花臂尾轮虫, Florida 品系 & 佛罗里达州一个季节性池塘 & 具有非常显著的延迟策略 & 否 & {$[44]$} \\
\hline 蓦花臂尾轮虫, Georgia 品系 & 佐治亚州一个永久性湖泊 & 不具有延迟策略 & 否 & {$[27]$} \\
\hline 角突臂尾轮虫, Argentina 品系 & 阿根廷一个季节性池塘 & 具有显著的延迟策略 & 否 & {$[27]$} \\
\hline 椎尾水轮虫品系一 & $\begin{array}{l}\text { 德国奥德河季节性洪水造成 } \\
\text { 的一个河漫滩 }\end{array}$ & 具有显著的延迟策略 & 否 & {$[27]$} \\
\hline 椎尾水轮虫品系二 & $\begin{array}{l}\text { 距离上述河漫滩约 } 17 \mathrm{~km} \text { 的 } \\
\text { 一个永久性湖泊 }\end{array}$ & 具有非常显著的延迟策略 & 是 & {$[27]$} \\
\hline 前额犀轮虫品系一 & $\begin{array}{l}\text { 德国奥德河季节性洪水造成 } \\
\text { 的一个河漫滩 }\end{array}$ & 具有非常显著的延迟策略 & 否 & {$[27]$} \\
\hline 前额犀轮虫品系二 & $\begin{array}{l}\text { 距离上述河漫滩约 } 17 \text { km 的 } \\
\text { 一个永久性湖泊 }\end{array}$ & 不具有延迟策略 & 否 & {$[27]$} \\
\hline 一种六腕轮虫种类 & $\begin{array}{l}\text { 北美洲 Chihuahuan 沙漠里小 } \\
\text { 型临时性水塘 }\end{array}$ & 不具有延迟策略 & 是 & {$[52]$} \\
\hline
\end{tabular}

* 是否具有有性生殖延迟策略的判断均基于原始文献中的统计分析.

\section{4 其他潜在的母系效应影响}

母系效应 (maternal effect) 通常是指子代的表型不仅仅由其基因型和发育过程中经历的环境条件所决 定, 还由母体的表型或经历的环境条件所决定的一种现象 ${ }^{[87]}$. 经过多年更为广泛的研究后人们发现母系效 应有着多种类型, 而它们通常有利于提高母体的适合度 ${ }^{[88]}$. 在很多无脊椎动物类群, 人们都发现母体经历的 环境条件可以显著的影响后代的生活史特征, 如在飞蝗中人们发现经历高种群密度环境的母体产下的后代 将具有显著更大的体型,而这种表型有利于提高后代面临相似环境时的种内竞争能力 ${ }^{[89]}$.

在浮游动物中, 一些研究也发现母系效应可以对子代的生活史对策产生显著影响. 例如, 在水蚤中人们 发现当子代 $\left(F_{1}\right)$ 的环境中食物条件与母体 $\left(F_{0}\right)$ 生活环境中的食物条件不一致时 (高 $\rightarrow$ 低/低 $\rightarrow$ 高), 特别是 当子代所处环境中食物条件恶化时 (高一低), 子代将改变用于繁殖的资源分配, 倾向于产生较高比例的有 性个体 $\left(\mathrm{F}_{2}\right)$; 这证明在水蚤中母体可以将其经历的环境信息传递给后代,这被称为 “代间记忆” (generational

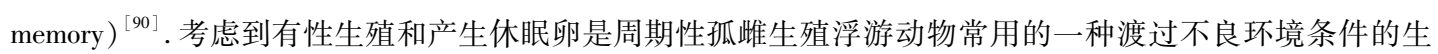
活史对策, 显然这种特殊的母系效应 ( “代间记忆”) 对于某一基因型的长期适合度是有利的. 但是在轮虫 中, 类似的母系效应是否会影响个体在有性生殖和孤雌生殖之间的资源分配并进一步影响整个种群的有性 生殖倾向性, 还有待进一步实验的证实.

进一步深人研究母系效应对轮虫有性生殖的影响还能够对揭示轮虫有性生殖机理 (详见本文前面相关 部分的论述) 有所帮助. 目前关于单巢类轮虫种群有性生殖发生的假说 (依据研究最为透彻的臂尾轮虫属种 类) 认为卵母细胞在母体体内的发育过程中母体生存环境中有性生殖诱导信号的浓度 (即密度信号) 决定了 子代中有性个体的比例 ${ }^{[4,19]}$. 同时, 关于卵母细胞发育过程中接受环境中有性生殖诱导信号从而决定后代发 育类型的信号通路, 目前有两个假说较为合理. 第一个假说认为体外的有性生殖诱导信号分子穿越轮虫的 体壁, 直接作用于卵母细胞, 并导致卵母细胞发育过程中的特定基因表达发生改变, 从而产生有性雌体; 第 二个假说认为体细胞上的受体接受到体外的信号分子后通过神经系统或是其他体内的信息传递方式将有 性生殖的诱导信号传递至生殖腺和生殖细胞, 从而间接影响卵母细胞的发育过程 ${ }^{[20]}$, 但在单巢类轮虫中具 体是采取哪种信号转导方式, 迄今尚无实证支持. 理论上, 如果母系效应对轮虫有性生殖的影响得到证实的 话 (例如发现类似于水蚤中 “代间记忆” 的效应), 那么至少可以证实上述第二种信号传递途经是存在的, 因 为如果仅存在第一种途径, 那么母体 $\left(F_{1}\right)$ 从上代 $\left(F_{0}\right)$ 获得的信息将无法影响到体内卵母细胞的发育过程, 因而 $F_{0}$ 代经历的环境状况 (环境中密度信号的浓度) 与 $F_{2}$ 代产生后代的有性个体比率无关. 


\section{8 性比率进化理论与单巢类轮虫的有性生殖策略}

\section{1 单巢类轮虫中均等雌雄比率的证据}

单巢类轮虫特殊的生活史决定了它们的有性生殖性别资源分配中存在一种特殊的负反馈机制: 如果环 境中的雄性个体稀少, 那么混交雌体受精的概率就很低, 这时它们就会生产雄卵并产出雄性个体; 而随着环 境中雄性个体密度的上升, 越来越多的幼年混交雌体受精并产生休眠卵, 从而种群中雄体的产量下降, 这种 负反馈机制就保证了种群性比率的稳定 ${ }^{[91]}$. 这里必须要着重指出的是, 在一般的性比率进化分析中, 研究对 象往往是种群中雌雄个体的比例, 而由于轮虫具有特殊的雌雄决定机制, 因而在轮虫中性比率进化分析的 研究对象应改为种群中生产雄性的有性雌体和生产休眠卵的有性雌体, 前者相当于一般进化意义上的雄 性,而后者相当于一般进化意义上的雌性 ${ }^{[92]}$.

在单巢类轮虫生活的水域环境中, 尽管各种资源 (例如食物) 的分布通常是呈现出一定区域异质性 (即 不同程度的斑块状分布), 但同时也表现出高度的流动性和扩散性 ${ }^{[93]}$, 同时研究发现轮虫有性生殖时的雌 雄相遇是随机的 ${ }^{[48,94]}$, 因此上述局域配偶竞争理论和局域资源竞争理论在轮虫性比率进化研究中并不适 用. 换句话说, 单巢类轮虫是一类适合 Fisher 模型的类群, 理论上它们种群的性比率 (在单巢类轮虫中, 此概 念指种群中生产雄性的有性雌体数目与种群中有性雌体总数的比率) 应接近 $0.5^{[91-92]}$.

尽管理论模型从进化稳定策略 (ESS) 的角度得出单巢类轮虫中应具有均等的雌雄比率, 但很长时间以 来一直缺乏直接的实验证据. Aparici 等 ${ }^{[92]}$ 设计了室内和室外两组实验 (共 4 个) 验证此假说. 结果表明两组 室内培养实验的结果均支持上述假说, 而对一个室外人工池塘中轮虫种群连续两年的监测结果中有一年的 数据并不支持此假说. 关于其中一年 (1996-1997 年) 的野外监测数据不支持均等的性比率假说, Aparici 等 给出了两条解释:一是 “单巢类轮虫中具有均等的性比率” 假说可能并不能局限在一个生长季内实现, 当综 合计算多个生长季内的性别资源分配情况时会得到支持此假说的结论; 二是环境条件的波动选择可能在不 同生长季内偏好不同的可受精年龄阈值 (详见下节论述), 而轮虫的这个特征则可以影响性比率, 同时环境 条件波动选择的压力将维持同域共存的不同基因型在这个特征上具有一定程度的多样性. 事实上, 如果仅 按时间计算, 则生长季内的大多数时段都是偏雄性的性比 (即性比率 $>0.5$ ), 只有在种群密度最高的一段时 间出现偏雌性的性比率, 考虑到此时巨大的种群规模作为基数, 才得到整个生长季累计种群具有均等的性 比率. 而野外观察已经证实淡水湖泊中轮虫种群动态的年际变化显著 ${ }^{[95]}$, 如果轮虫种群在某一生长季内的 种群密度显著低于多年平均的峰值密度, 那么理论上急剧且显著偏雌的性比率将难以出现. 这样将导致按 照整个生长季计算的种群性比率将显著偏雄性. 所以, 笔者认为均等的性比率对于一个单巢类轮虫种群来 说应该是一个长期的概念, 而在某一个生长季内种群的性比率是可以显著背离 0.5 的.

\section{2 单巢类轮虫种群性比率的影响因素}

通过对单巢类轮虫特殊的生活史进行详细研究后人们发现其种群的性比率受到两个因素的直接影响: 一是有性雌体的可受精时间阈值 (在轮虫中此概念的定义是有性雌体可以与雄体成功交配且受精的最大年 龄, 如果有性雌体在此年龄之内未成功交配, 则成熟后将产生雄卵; 而如果其在此年龄之内成功交配, 则成 熟后将产生休眠卵; Threshold Age of Fertilization, TAF). 二是种群中的雄性个体密度 ${ }^{[91]}$. 理论上说, 如果轮虫 的有性个体能够调节其 TAF, 那么对其适合度有利的调节是: 当种群密度高时 (此时往往也意味着具有较高 的雄体密度), 有性雌体遇到雄体的概率也较大, 因而应该缩短 TAF; 当种群密度低时 (此时往往也意味着具 有较低的雄体密度), 有性雌体遇到雄体的概率也较小, 因而应该延长 TAF 以提高受精的概率, 即有性雌体 的 TAF 与有性雌体密度呈现显著的负相关 ${ }^{[96]}$. 利用已知的禇皱臂尾轮虫的生活史参数, Aparici 等 ${ }^{[91]}$ 采用 ESS 可侵人性模型的方法研究了最佳 TAF 的问题. 结果表明在不同的环境条件下 (这直接影响了种群具有 不同的出生率、死亡率等生活史参数, 同时高的种群密度也意味着雌雄相遇概率较高), 最适 TAF 不同: 当环 境条件很适宜时, 种群倾向于较低的 TAF; 相反, 在环境条件恶劣时, 种群 ESS 的 TAF 较高. 在不同环境下, 禇皱臂尾轮虫最佳 TAF 的范围在其成熟年龄的 $14.2 \% \sim 29.2 \%$ 之间, 即 TAF 总是显著小于个体的成熟年 龄, 这个结论也与实际观察的结果相吻合 ${ }^{[97-98]}$.

\section{9 小结与展望}

本文较系统地综述了国内外轮虫研究在有性生殖机制与进化策略方面的最新进展, 主要包括单巢类轮 
虫有性生殖的机理、理论模型、影响有性生殖发生和有性生殖策略的内、外因素以及性比率理论在轮虫有性 生殖研究中的应用等几个方面. 笔者认为, 当前轮虫研究在单巢类轮虫有性生殖领域还存在以下几个亟待 深人研究的薄弱环节:

第一, 尽管目前很多证据都暗示固醇类物质是单巢类有性生殖的关键信息素, 但是由于过去水体中信 息素物质分析技术和结构生物学研究手段的局限,这类物质在轮虫体内的作用机理 (信号通路)并未真正阐 明. 现在随着研究方法和技术的进步, 揭示决定单巢类轮虫有性生殖策略的关键信号通路越来越成为可能.

第二, 目前的证据支持在单巢类轮虫的很多类群中, 延迟的有性生殖策略是一种广泛存在的现象. 但目 前的 ESS 可侵人模型和实证研究都未能够很好的论证这种有性生殖策略有多大的适应意义, 特别是在长期 适合度方面. 另外, 轮虫在很多生活史或是表型方面都有很强的可塑性, 那么这种重要的生殖策略本身是否 具有相当程度上的可塑性? 对于没有这种策略的轮虫物种或是基因型, 它们是通过何种机制与具有这种策 略的物种或基因型长期共存的?

第三,在过去数十年里, 人们基于资源竞争理论 ${ }^{[99]}$ 和生态位理论 ${ }^{[100]}$ 做了大量轮虫类种间或是隐种间 的竞争与共存研究. 然而最近的理论研究发现在周期性孤䧳生殖的两个物种间, 即使没有任何生态位分化 而仅仅依赖有性生殖策略的不同即可实现稳定共存 ${ }^{[101]}$,但目前这个理论的实验或观测证据还极端缺乏.

\section{0 参考文献}

[ 1 ] Wallace RL, Snell TW. Phylum rotifera. In: Thorp JH, Covich AP eds. Ecology and classification of North American freshwater invertebrates(Second Edition). California: Academic Press, 2001 : 195-254.

[ 2 ] Segers H. Annotated checklist of the rotifers (Phylum Rotifera), with notes on nomenclature, taxonomy and distribution. Zootaxa, 2007, 1564: 1-104.

[ 3 ] Gilbert JJ. Contact chemoreception, mating behaviour, and sexual isolation in the rotifer genus Brachionus. Journal of Experimental Biology, 1963, 40(4) : 625-641.

[ 4 ] Snell TW, Kubanek J, Carter W et al. A protein signal triggers sexual reproduction in Brachionus plicatilis (Rotifera). Marine Biology, 2006, 149: 763-773.

[ 5 ] Kubanek J, Snell TW. Quorum sensing in rotifers. In: Winans SC, Bassler BL eds. Chemical communication among bacteria. Washington: ASM Press, 2008: 453-461.

[ 6 ] Snell T, Rico-Martinez R, Kelly L et al. Identification of a sex pheromone from a rotifer. Marine Biology, 1995, 123: 347-353.

[ 7 ] Snell T, Stelzer CP. Removal of surface glycoproteins and transfer among Brachionus species. Hydrobiologia, 2005, 546: 267-274.

[ 8 ] Fussmann GF, Ellner SP, Hairston NG. Evolution as a critical component of plankton dynamics. Proceedings of the Royal Society of London. Series B : Biological Sciences, 2003, 270 : 1015-1022.

[ 9 ] Snell T, Shearer T, Smith H et al. Genetic determinants of mate recognition in Brachionus manjavacas (Rotifera). BMC Biology, 2009, 7(1): 60 .

[10] Gallardo WG, Hagiwara A, Tomita Y et al. Effect of some vertebrate and invertebrate hormones on the population growth, mictic female production, and body size of the marine rotifer Brachionus plicatilis Müller. Hydrobiologia, 1997, 358: 113-120.

[11] Gallardo WG, Hagiwara A, Snell TW. Effect of juvenile hormone and serotonin (5-HT) on mixis induction of the rotifer Brachionus plicatilis Müller. Journal of Experimental Marine Biology and Ecology, 2000, 252 : 97-107.

[12] Snell TW, DesRosiers NJD. Effect of progesterone on sexual reproduction of Brachionus manjavacas (Rotifera). Journal of Experimental Marine Biology and Ecology, 2008, 363(1/2) : 104-109.

[13] 夏继刚, 牛翠娟. 壬基酚暴露对斑马鱼求偶行为与繁殖成功率的影响. 生态学报, 2010, 30(20): 5537-5543.

[14] Preston BL, Snell TW, Robertson TL et al. Use of freshwater rotifer Brachionus calyciflorus in screening assay for potential endocrine disruptors. Environmental Toxicology and Chemistry, 2000, 19(12) : 2923-2928.

[15] Snell TW, Joaquim-Justo C. Workshop on rotifers in ecotoxicology. Hydrobiologia, 2007, 593 (1) : 227-232.

[16] Schmitt A, Nebreda AR. Signalling pathways in oocyte meiotic maturation. Journal of Cell Science, 2002, 115 ( 12 ): 2457-2459.

[17] Gilbert JJ. Rotifera. In: Adiyodi KG, Adiyodi RG eds. Reproductive biology of invertebrates. Oogenesis, Oviposition, and Oosorption. New York: John Wiley \& Sons Ltd. , 1983: 181-209.

[18] Gilbert JJ. Induction of mictic females in the rotifer Brachionus: oocytes of amictic females respond individually to popula- 
tion-density signal only during oogenesis shortly before oviposition. Freshwater Biology, 2007, 52( 8 ) : 1417-1426.

[19] Snell TW. A review of the molecular mechanisms of monogonont rotifer reproduction. Hydrobiologia, 2011, 662 : 89-97.

[20] Gilbert JJ. Environmental and endogenous control of sexuality in a rotifer life cycle: developmental and population biology. Evolution and Development, 2003, 5(1): 19-24.

[21] Serra M, King CE. Optimal rates of bisexual reproduction in cyclical parthenogens with density-dependent growth. Journal of Evolutionary Biology, 1999, 12(2) : 263-271.

[22] Pourriot R, Snell TW. Resting eggs in rotifers. Hydrobiologia, 1983, 104: 213-224.

[23] Snell TW, Boyer EM. Thresholds for mictic female production in the rotifer Brachionus plicatilis (Müller). Journal of Experimental Marine Biology and Ecology, 1988, 124(2) : 73-85.

[24] Stelzer CP, Snell TW. Induction of sexual reproduction in Brachionus plicatilis (Monogononta, Rotifera) by a density-dependent chemical cue. Limnology and Oceanography, 2003, 48(2) : 939-943.

[25] Gilbert JJ. Specificity of crowding response that induces sexuality in the rotifer Brachionus. Limnology and Oceanography, 2003, $48(3)$ : 1297-1303.

[26] Gilbert JJ, Dieguez MC. Low crowding threshold for induction of sexual reproduction and diapause in a Patagonian rotifer. Freshwater Biology, 2010, 55(8) : 1705-1718.

[27] Schröder T, Gilbert JJ. Transgenerational plasticity for sexual reproduction and diapause in the life cycle of monogonont rotifers: intraclonal, intraspecific and interspecific variation in the response to crowding. Functional Ecology, 2004,18 (3) : $458-466$.

[28] Timmermeyer N, Stelzer CP. Chemical induction of mixis in the rotifer Synchaeta tremula. Journal of Plankton Research, 2006, $28(12):$ 1233-1239.

[29] Carmona MJ, Gómez A, Serra M. Mictic patterns of the rotifer Brachionus plicatilis Müller in small ponds. Hydrobiologia, 1995, 313/314: 365-371.

[30] Larsson P, Dodson S. Invited review: chemical communication in planktonic animals. Archiv für Hydrobiologie, 1993, 129(2) : 129-155.

[31] Fitzsimmons JM, Innes DJ. Inter-genotype variation in reproductive response to crowding among Daphnia pulex. Hydrobiologia, 2006, 568: 187-205.

[32] Gilbert JJ. Timing of diapause in monogonont rotifers: mechanisms and strategies. In: Alekseev V, Stasio B, Gilbert J eds. Diapause in aquatic invertebrates: Theory and human use. Dordrecht, Netherlands: Springer, 2007: 11-27.

[33 ] Serra M, Smith H, Weitz J et al. Analysing threshold effects in the sexual dynamics of cyclically parthenogenetic rotifer populations. Hydrobiologia, 2011, 662: 121-130.

[34] Gilbert JJ, Thompson GA. Alpha tocopherol control of sexuality and polymorphism in the rotifer Asplanchna. Science, $1968,159(3816)$ : 734-736.

[35] Gilbert JJ. Female polymorphism and sexual reproduction in the rotifer Asplanchna: evolution of their relationship and control by dietary tocopherol. American Naturalist, 1980, 116(3) : 409-431.

[36 ] Pourriot R, Clément P. Influence de la durée de l'éclairement quotidien sur le taux de femelles mictiques chez Notommata copeus Ehr. (Rotifère). Oecologia, 1975, 22 (1) : 67-77.

[37] Gilbert J. Mictic-female production in monogonont rotifers. Archiv Hydrobiologie Beiheft, 1977, 8: 142-155.

[38 ] Gilbert JJ. Dormancy in rotifers. Transactions of the American Microscopical Society, 1974, 93 : 490-513.

[39] Serra M, Snell TW, Gilbert JJ. Delayed mixis in rotifers: an adaptive response to the effects of density-dependent sex on population growth. Journal of Plankton Research, 2005, 27 (1) : 37-45.

[40] Gilbert JJ. Mictic female production in the rotifer Brachionus calyciflorus. Journal of Experimental Zoology, 1963,153 (2) : 113-123.

[41] Hobæk A, Larsson P. Sex determination in Daphnia magna. Ecology, 1990, 71(6) : 2255-2268.

[42] Gilbert JJ, Walsh EJ. Brachionus calyciflorus is a species complex : mating behavior and genetic differentiation among four geographically isolated strains. Hydrobiologia, 2005, 546: 257-265.

[43] Stelzer CP, Snell TW. Specificity of the crowding response in the Brachionus plicatilis species complex. Limnology and Oceanography, 2006, 51(1): 125-130.

[44] Gilbert JJ. Endogenous regulation of environmentally induced sexuality in a rotifer: a multigenerational parental effect induced by fertilisation. Freshwater Biology, 2002, 47(9): 1633-1641.

[45] Gómez A, Snell TW. Sibling species and cryptic speciation in the Brachionus plicatilis species complex (Rotifera). Jour- 
nal of Evolutionary Biology, 1996, 9(6) : 953-964.

[46] Snell TW. Sex, population dynamics and resting egg production in rotifers. Hydrobiologia, 1987, 144: 105-111.

[47] Serra M, Snell TW, King CE. The timing of sex in cyclically parthenogenetic rotifers. In: Moya A, Font E eds. Evolution: from molecules to ecosystems. New York: Oxford University Press, 2004: 135-146.

[48 ] Snell TW, Garman BL. Encounter probabilities between male and female rotifers. Journal of Experimental Marine Biology and Ecology, 1986, 97(3): 221-230.

[49] Ciros-Pérez J, Carmona MJ, Serra M. Resource competition and patterns of sexual reproduction in sympatric sibling rotifer species. Oecologia, 2002, 131(1) : 35-42.

[50] Gilbert JJ. Effect of food concentration on the production and viability of resting eggs of the rotifer Brachionus: implications for the timing of sexual reproduction. Freshwater Biology, 2010, 55 : 2437-2446.

[51] Serra M, Carmona MJ. Mixis strategies and resting eeg production of rotifers living in temporally-varying habitats. Hydrobiologia, 1993, 255 : 117-126.

[52] Schröder T, Howard S, Arroyo M et al. Sexual reproduction and diapause of Hexarthra sp. (Rotifera) in short lived ponds in the Chihuahuan Desert. Freshwater Biology, 2007, 52: 1033-1042.

[53] Hino A, Hirano R. Ecological studies on the mechanism of bisexual reproduction in the rotifer Brachionus plicatilis. II . Effects of cumulative partenogenetic generation on the frequency of bisexual reproduction. Bulletin of the Japanese Society of Scientific Fisheries, 1977, 43 : 1147-1155.

[54] Merriman JL, Kirk KL. Temporal patterns of resource limitation in natural populations of rotifers. Ecology, 2000, 81 (1) : 141-149.

[55] Snell TW, Carrillo K. Body size variation among strains of the rotifer Brachionus plicatilis. Aquaculture, 1984,37 (4) : 359-367.

[56] King CE, Snell TW. Density-dependent sexual reproduction in natural populations of the rotifer Asplanchna girodi. Hydrobiologia, 1980, 73 : 149-152.

[57] Snell TW, Hoff FH. The effect of environmental factors on resting egg production in the rotifer Brachionus plicatilis. Journal of the World Mariculture Society, 1985, 16(4) : 484-497.

[58] Snell TW, Hoff FH. Fertilization and male fertility in the rotifer Brachionus plicatilis. Hydrobiologia, 1987, 147: 329-334.

[59] Hagiwara A, Hino A, Hirano R. Studies on the formation and hatching of fertilized eggs of the rotifer Brachionus plicatilis. 2. Effects of temperature and chlorinity on resting egg formation. Nippon Suisan Gakkaishi, 1988, 54(4) : 569-575.

[60] Pourriot R, Rougier C. Température, démographie et mixis chez un rotifère héléoplanctonique, Epiphanes brachionus ( Ehrb. ). Annales de Limnologie, 1999, 35: 167-172.

[61] Montero-Pau J, Ramos-Rodríguez E, Serra M et al. Long-term coexistence of rotifer cryptic species. Plos One, 2011, 6: e21530.

[62 ] Lubzens E, Minkoff G, Marom S. Salinity dependence of sexual and asexual reproduction in the rotifer Brachionus plicatilis. Marine Biology, 1985, 85(2) : 123-126.

[63 ] Snell TW. Effect of temperature, salinity and food level on sexual and asexual reproduction in Brachionus plicatilis ( Rotifera). Marine Biology, 1986, $92(2): 157-162$.

[64] Hagiwara A, Lee CS, Miyamoto G et al. Resting egg formation and hatching of the S-type rotifer Brachionus plicatilis at varying salinities. Marine Biology, 1989, 103(3) : 327-332.

[65] Hagiwara A, Hamada K, Hori S et al. Increased sexual reproduction in Brachionus plicatilis (Rotifera) with the addition of bacteria and rotifer extracts. Journal of Experimental Marine Biology and Ecology, 1994, 181(1) : 1-8.

[66] 沈蕴芬. 水细菌生态. 见: 刘建康编. 高级水生生物学. 北京: 科学出版社, 1999: 151-175.

[67] Lansing AI. A transmissible, cumulative, and reversible factor in aging. Journal of Gerontology, 1947, 2 (3) : 228-239.

[68] Lansing AI. A nongenic factor in the longevity of rotifers. Annals of the New York Academy of Sciences, 1954,57 ( 5) : $455-464$.

[69] Parsons PA. Parental age and the offspring. Quarterly Review of Biology, 1964, 39(3) : 258-275.

[70] Schröder T, Gilbert JJ. Maternal age and spine development in the rotifer Brachionus calyciflorus: increase of spine length with birth orders. Freshwater Biology, 2009, 54(5): 1054-1065.

[71] Kim HJ, Hagiwara A. Effect of female aging on the morphology and hatchability of resting eggs in the rotifer Brachionus plicatilis Müller. Hydrobiologia, 2011, 662 : 107-111. 
[ 72 ] Pourriot R, Rougier C. Influence de l'âge des parents sur la production de femelles mictiques chez Brachionus calyciflorus (Pallas) et B. rubens Ehr. (Rotifères). Comptes Rendus de l’Académie des Sciences Paris, 1976, 283 : 1497-1500.

[ 73 ] Pourriot R, Rougier C. Effets de la densité de population et du groupement sur la reproduction de Brachionus calyciflorus (Pallas) (Rotifère). Annales de Limnologie, 1977, 13 : 101-113.

[ 74 ] Carmona MJ, Serra M, Miracle MR. Effect of population density and genotype on life-history traits in the rotifer Brachionus plicatilis O. F. Müller. Journal of Experimental Marine Biology and Ecology, 1994, 182(2) : 223-235.

[ 75 ] Pourriot R, Rougier C. Influences conjuguées du groupement et de la qualité de la nourriture sur la reproduction de Brachionus plicatilis O. F. Müller (Rotifère). Netherlands Journal of Zoology, 1979, 29: 242-264.

[ 76 ] Gilbert JJ, Schröder T. Intraclonal variation in propensity for mixis in several rotifers: variation among females and with maternal age. Hydrobiologia, 2007, 593: 121-128.

[ 77 ] Lubzens E, Minkoff G. Influence of the age of algae fed to rotifers (Brachionus plicatilis O. F. Müller) on the expression of mixis in their progenies. Oecologia, 1988, 75(3): 430-435.

[ 78 ] Fussmann GF, Kramer G, Labib M. Incomplete induction of mixis in Brachionus calyciflorus : patterns of reproduction at the individual level. Hydrobiologia, 2007, 593: 111-119.

[ 79 ] Gilbert JJ, Schröder T. Rotifers from diapausing, fertilized eggs: Unique features and emergence. Limnology and Oceanography, 2004, 49(4): 1341-1354.

[ 80 ] Hagiwara A, Kadota Y, Hino A. Maternal effect by stem females in Brachionus plicatilis : effect of starvation on mixis induction in offspring. Hydrobiologia, 2005, 546: 275-279.

[ 81 ] Hairston NG. Zooplankton egg banks as biotic reservoirs in changing environments. Limnology and Oceanography, 1996, $41(5)$ : 1087-1092.

[ 82 ] Maynard SJ, Price GR. The logic of animal conflict. Nature, 1973, 246(5427) : 15-18.

[ 83 ] Miracle MR, Serra M. Salinity and temperature influence in rotifer life history characteristics. Hydrobiologia, 1989, 186 : $81-102$.

[ 84 ] Brendonck L, De Meester L. Egg banks in freshwater zooplankton: evolutionary and ecological archives in the sediment. Hydrobiologia, 2003, 491 : 65-84.

[ 85 ] Liu W, Niu CJ. Polymorphism in resting egg size and hatching strategy in the rotifer Brachionus calyciflorus Pallas. Zoological Science, 2010, 27 (4) : 330-337.

[ 86 ] Sommer U. Plankton ecology : succession in plankton communities. New York: Springer-Verlag, 1989.

[ 87 ] Mousseau TA, Fox CW. Maternal effects as adaptations. New York: Oxford University Press, 1998.

[ 88 ] Marshall DJ, Uller T. When is a maternal effect adaptive? Oikos, 2007, 116(12) : 1957-1963.

[ 89 ] Chapuis M, Crespin L, Estoup A et al. Parental crowding influences life-history traits in Locusta migratoria females. Bulletin of Entomological Research, 2010, 100 (1) : 9-17.

[ 90 ] LaMontagne JM, McCauley E. Maternal effects in Daphnia: what mothers are telling their offspring and do they listen? Ecology Letters, 2001, 4(1): 64-71.

[ 91 ] Aparici E, Carmona MJ, Serra M. Sex allocation in haplodiploid cyclical parthenogens with density-dependent proportion of males. American Naturalist, 1998, 152(4) : 652-657.

[ 92 ] Aparici E, Carmona MJ, Serra M. Evidence for an even sex allocation in haplodiploid cyclical parthenogens. Journal of Evolutionary Biology, 2002, 15(1): 65-73.

[ 93 ] Reynolds CS. Ecology of phytoplankton. New York: Cambridge University Press, 2006.

[ 94 ] Gómez A, Serra M. Mate choice in male Brachionus plicatilis rotifers. Functional Ecology, 1996, 10: 681-687.

[ 95 ] González MJ, Frost TM. Food limitation and seasonal population declines of rotifers. Oecologia, 1992, 89(4) : 560-566.

[ 96 ] Serra M, Aparici E, Carmona MJ. When to be sexual: sex allocation theory and population density-dependent induction of sex in cyclical parthenogens. Journal of Plankton Research, 2008, 30(11) : 1207-1214.

[ 97 ] Snell T, Childress M. Aging and loss of fertility in male and female Brachionus plicatilis (Rotifera). International Journal of Invertebrate Reproduction and Development, 1987, 12(1) : 103-110.

[98 ] 席贻龙, 黄祥飞. 荣花臂尾轮虫交配行为和受精作用研究. 生态学报, 2000, 20(4) : 541-544.

[ 99 ] Tilman D. Resource competition and community structure. Princeton: Princeton University Press, 1982.

[100 ] Colwell RK, Futuyma DJ. On the measurement of niche breadth and overlap. Ecology, 1971, 53(4) : 567-576.

[101] Montero-Pau J, Serra M. Life-cycle switching and coexistence of species with no niche differentiation. Plos One, 2011, 6: e20314. 